Galactic \& Stellar Dynamics

C. Boily, P. Patsis, S. Portegies Zwart, R. Spurzem and C. Theis (eds)

EAS Publications Series, 10 (2003) 1-2

\title{
ERRATUM: GAS DYNAMICS IN CENTRAL PARTS OF GALAXIES
}

\begin{abstract}
W. Maciejewski ${ }^{1}$
An error occurred during the compilation of the proceedings of the workshop Galactic and Stellar Dynamics. The final version of Figure 1 in the invited review by Witold Maciejewski was not forwarded as intended to the publisher, EDP Science. Instead, a draft version went to press. The error was not spotted until the book had been printed.

We apologize to the author for this mistake, for which we accept full responsibility. The correct Figure 1 is reproduced here along with its caption, as it improves significantly the clarity of the arguments. An edited version of the review was posted by the author at the Los Alamos archives site (reference astro-ph/0302250) prior to the publication of this volume.
\end{abstract}

The Editors

\footnotetext{
1 INAF - Osservatorio Astrofisico di Arcetri, Largo E. Fermi 5, 50125 Firenze, Italy, and Obserwatorium Astronomiczne Uniwersytetu Jagiellońskiego, Cracow, Poland

(C) EAS, EDP Sciences 2003 DOI: $10.1051 /$ eas:2003160
} 


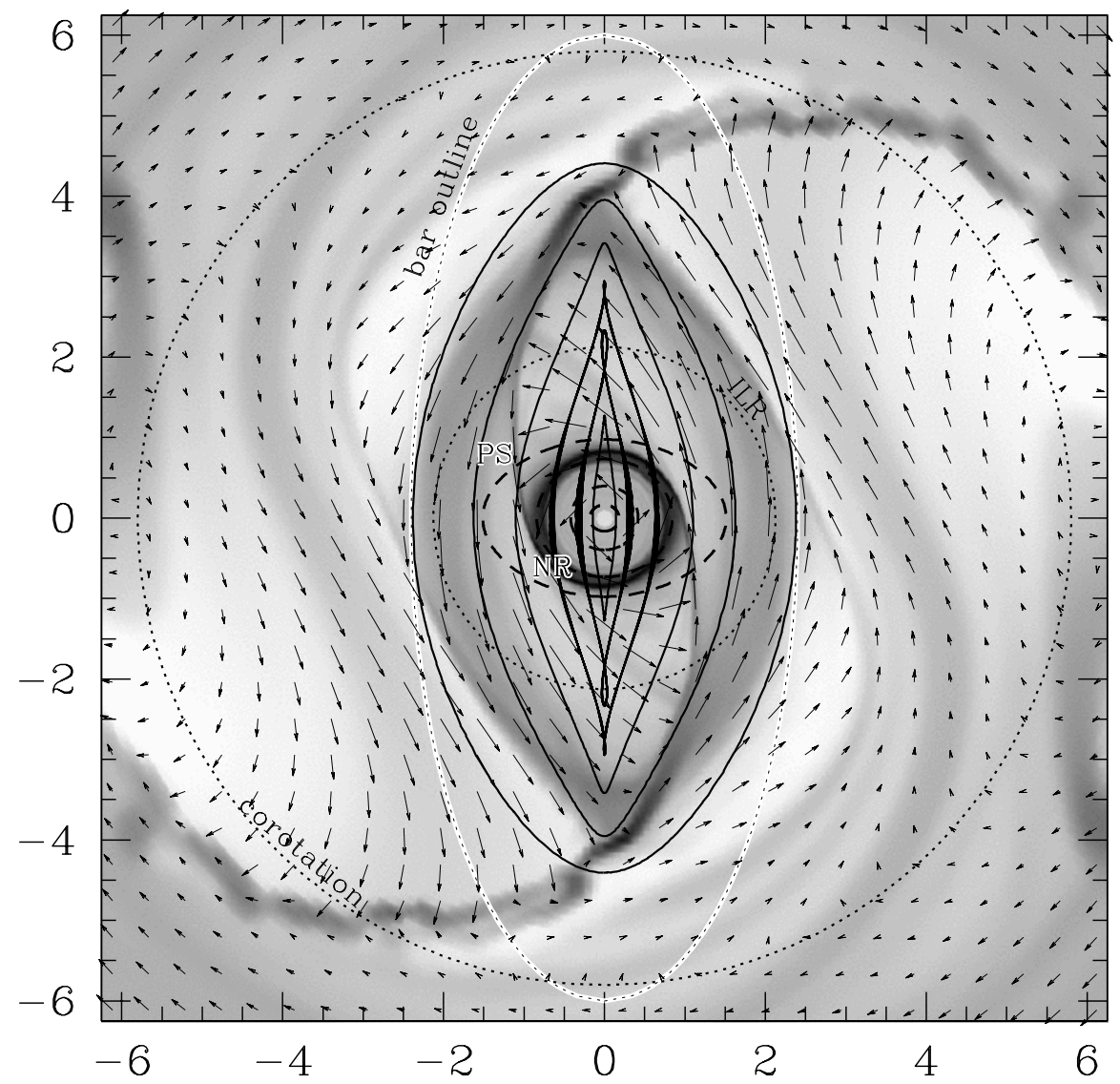

Fig. 1. A representative snapshot of the gas density and velocity field in a barred galaxy, taken after 6 rotation periods of the bar, once the main flow patterns have been established. The gas, treated as a non-selfgravitating, isothermal fluid with a sound speed of $5 \mathrm{~km} \mathrm{~s}^{-1}$, responds to a fixed gravitational potential of a bar, disc and spheroid, and is modeled with an Eulerian code on a fixed grid. The density is shown in grayscale, and arrows mark gas velocity in the reference frame rotating with the bar. Dotted circles mark corotation and the ILR. Examples of $x_{1}$ and $x_{2}$ orbits are drawn with solid and dashed lines, respectively. PS marks the principal shock in the bar, NR is the nuclear ring. Units on axes are in kpc. From the model S05 by Maciejewski et al. (2002). 